\title{
Video Mediastinoscopically Assisted Tracheal Release in Treatment of Long Tracheal Stenosis
}

\author{
Andreas Kirschbaum ${ }^{1}$ Afsin Teymoortash ${ }^{2}$ \\ ${ }^{1}$ Department of Surgery, University Hospital, Marburg, Germany \\ 2 Department of Head and Neck Surgery, University Hospital, Marburg, \\ Germany \\ Address for correspondence Andreas Kirschbaum, MD, Department \\ of Surgery, University Hospital, Baldingerstrasse, Marburg 35033. \\ Germany (e-mail: Kirschbaum001@gmx.de).
}

Thorac Cardiovasc Surg Rep 2013;2:32-34.
Abstract
Keywords
- trachea
- thoracic surgery
- mediastinum
- mediastinoscopy

The main prerequisite for the unimpaired wound healing after resection of tracheal stenosis is its tension-free end-to-end anastomosis. This is only achievable when the tracheal ends can be adequately mobilized. Several mobilization techniques have been described in the literature. Here, the authors describe a case of tracheal mobilization under video mediastinoscopic assistance that displayed a considerable advantage over blunt mobilization and a length gain of over $3 \mathrm{~cm}$.

\section{Introduction}

We treated a 53-year-old female complaining of worsening stridor and exertional dyspnoea so serious that she could no longer climb stairs. She presented no other known or relevant systemic comorbidities. Tracheoscopy revealed a concentric Cotton grade III subglottic stenosis approximately $5 \mathrm{~cm}$ long. The bronchial system distal of the stenosis was entirely inconspicuous. Wegener granulomatosis was excluded with tracheal mucosal biopsy and a blood test that looks for antineutrophil cytoplasmic antibodies.

The computed tomography scan of throat and thorax performed thereafter confirmed the stenosis, visible bronchoscopically without any other pathological anomalies. In response to this patient's obviously worsening clinical symptoms, a tracheal resection of the stenosis was recommended.

Although initiated with a laryngeal mask, the intervention proceeded under general anesthesia. Via a Kocher collar incision, we penetrated the thyroid isthmus, revealing the trachea and pharynx. The trachea's shape had not been changed by the stenosis. The trachea was mobilized with a finger ventral of the former, which only moved the trachea cranially by $1 \mathrm{~cm}$. Since the fingertip ended at the height of the brachiocephalic trunk, we could not mobilize the trachea more. A video mediastinoscope (Storz, Tuttlingen, Germany) was placed in front of

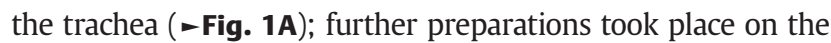
monitor ( - Fig. 1B). Using an isolated suction device, the trachea was freed bilaterally up to the bifurcation. Essential structures (i.e., the vena cava and central pulmonary artery) were shown and could thus be spared. Moreover, the primary bronchi was completely mobilized on both sides. With this technique it was possible to extend the trachea for additional $4 \mathrm{~cm}$.

The next step was marking the extent of the stenosis bronchoscopically via the laryngeal mask. We were surprised by its extent which was far longer than the endoscope had led us to believe. The trachea was opened beneath the stenosis and penetrated at that location. After applying a holding suture, the trachea was intubated with a $\mathrm{CH}$ 6.0 tube and continued ventilation. To resect the tracheostenosis completely, five tracheal rings had to be removed, corresponding to a resection length of $5.5 \mathrm{~cm}$. The posterior wall of the anastomosis was reconstructed with single-knot stitches (Vicryl 4-0). The anterior wall was connected with single-knot stitches (polydioxanone 2-0) after orotracheal intubation. After anteflexion of the head the result was an entirely airtight tension-free anastomosis (-Fig. 2).

At the end of the operation, we didn't fix the chin to the sternum, there was a preoperative commitment of the cooperative patient to anteflex the neck for 7 days after the operation. The patient was ventilated for a day and then extubated without any complications. Her further postoperative course was entirely uneventful, and she was released home 1 week after surgery. received

April 21, 2013

accepted after revision

June 12, 2013

published online

July 16, 2013
DOI http://dx.doi.org/ 10.1055/s-0033-1350104. ISSN 2194-7635. (c) 2013 Georg Thieme Verlag KG Stuttgart · New York
License terms

()(1) $\Theta \circledast$ 


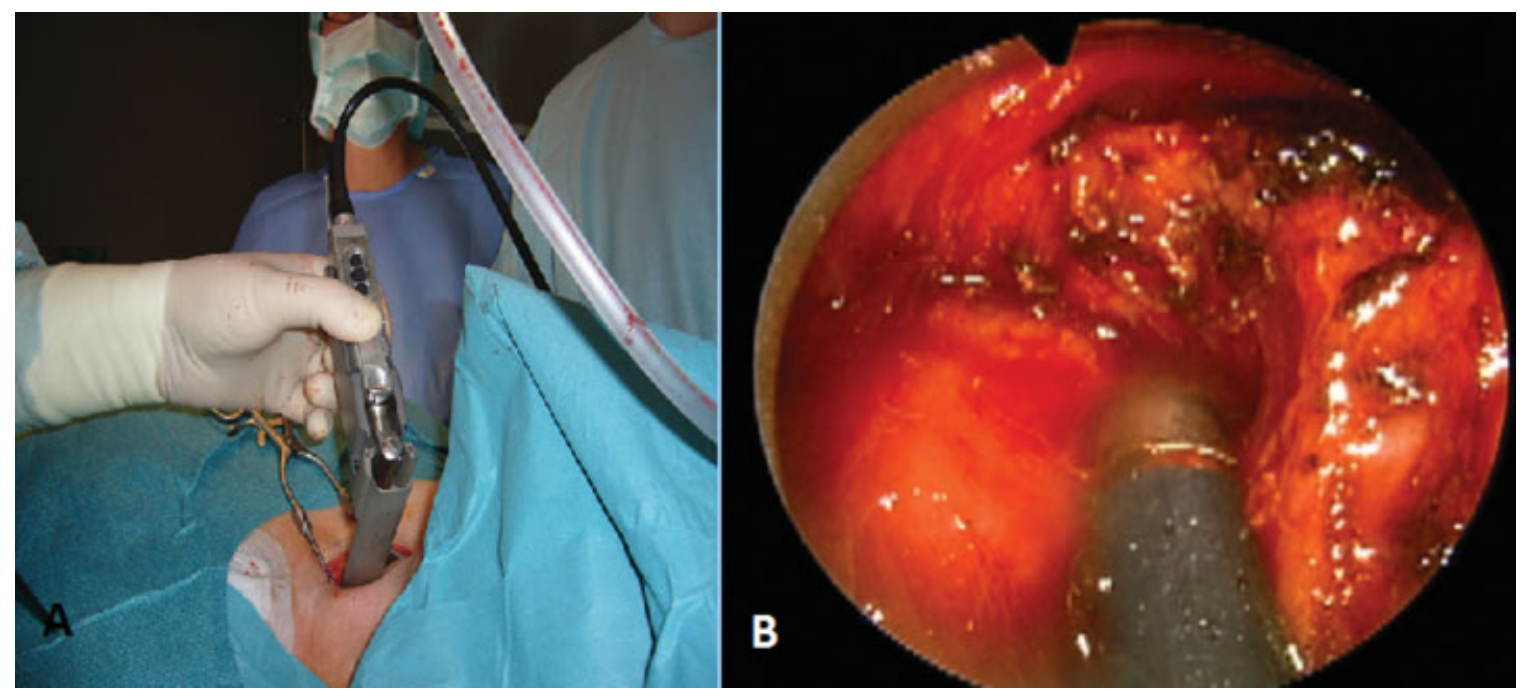

Fig. 1 (A) Video mediastinoscope in distal position. (B) Monitor image video mediastinoscopically assisted tracheal mobilization using an isolated succer.

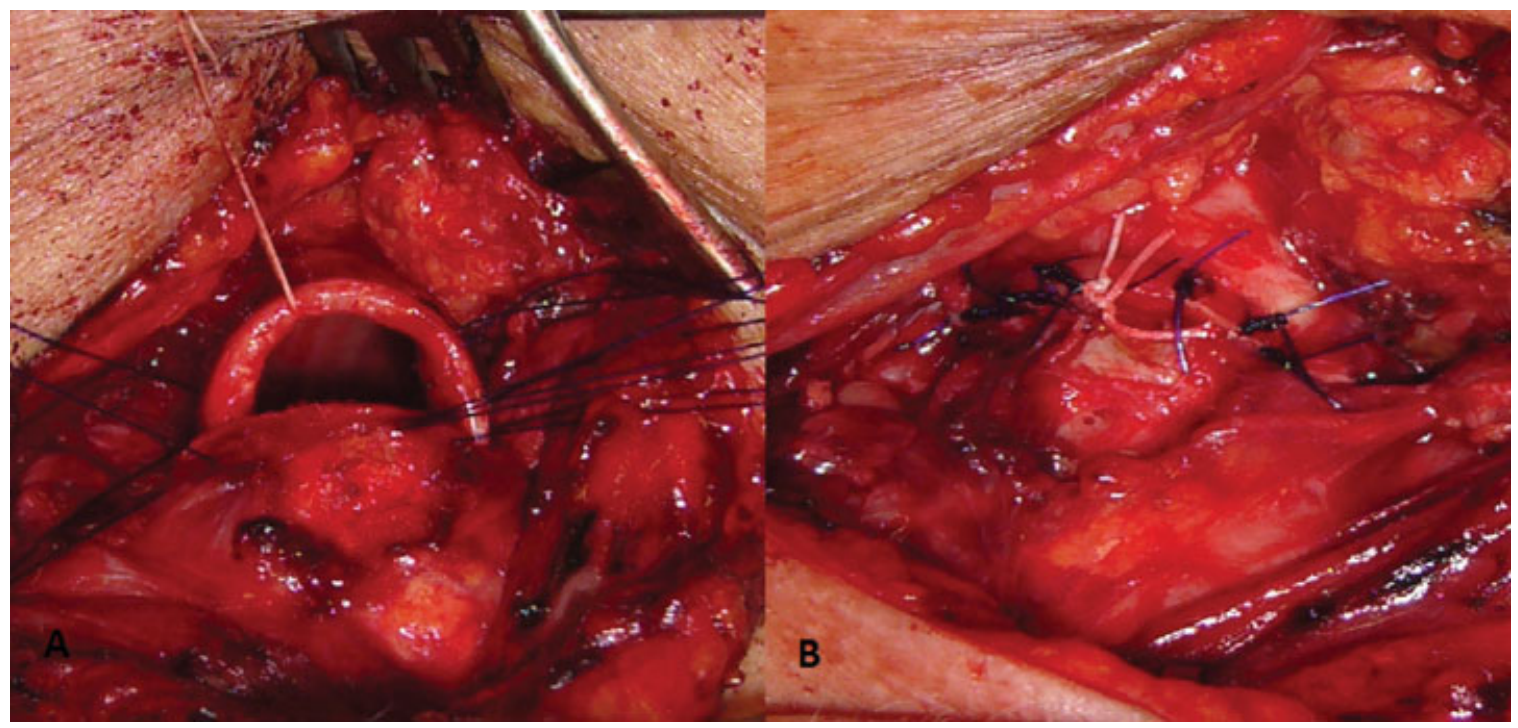

Fig. 2 Tracheal anastomosis (A) before and (B) after placement.

\section{Discussion}

One of the corner stones for the complication-free healing of a tracheal anastomosis is ensuring a position free of tension. The means of digitally mobilizing the trachea distally are very limited when approaching from the neck. Moreover, when the dissection is blind the risk of bleeding is serious and potentially difficult to manage. After reading about the positive results of Kang et al ${ }^{1}$ who used a mediastinoscope for resection of a lengthy tumorstenosis of the trachea, it was decided to employ a video mediastinoscope while resecting an idiopathic tracheal stenosis. Positioned in front of the trachea the video mediastinoscope made surgery under observation and the display of the most important peritracheal structures possible; it visualizes the vena cava and central pulmonary artery. Preparation can take place bilaterally as far as the distal main bronchus. Minor bleeding can be stemmed under observation with an isolated suction device. The trachea can thus be cranially mobilized by 3 to $4 \mathrm{~cm}$. There is some risk to impair the blood supply of the distal trachea by too much intensive bilateral mobilization, so the extensive preparation at the lateral areas of the distal trachea was avoided. We believe the additional often recommended suprahyoid release of the trachea ${ }^{2}$ can be avoided. Transthoracic hilar dissection as recommended by Maeda and Grillo ${ }^{3}$ by which the pulmonary ligament is transsected and an incision made into the pericardium is technically very demanding and only doable via an additional thoracic approach. Furthermore, only one side of the tracheobronchial tree can be mobilized. The present case demonstrates the considerable advantage of video mediastinoscopically assisted release in which both sides of the tracheobronchial tree can be mobilized without an additional approach.

\section{Conclusion}

Video mediastinoscopically assisted release is a simple method of risk-free mobilizing of the entire distal trachea and ensuring maximum length. We recommend it highly for extensive tracheal resections. 
34 Video Mediastinoscopically Assisted Tracheal Release Kirschbaum, Teymoortash

\section{References}

1 Kang JH, Park IK, Bae MK, Hwang Y. Mediastinoscopic bilateral bronchial release for long segmental resection and anastomosis of the trachea. Korean J Thorac Cardiovasc Surg 2011;44(3): 257-259
2 Montgomery WW. Tracheal stenosis. In: Montgomery WW, ed. Surgery of the Larynx, Trachea Esophagus and Neck. Philadelphia, PA: Saunders; 2001:234-239

3 Maeda M, Grillo HC. Effect of tension on tracheal growth after resection and anastomosis in puppies. J Thorac Cardiovasc Surg 1973;65(4):658-668 